\title{
Experimental study and modelling of heat transfer during anodizing in a wall-jet set-up
}

\author{
T. Aerts ${ }^{1}$, G. Nelissen ${ }^{2}$, J. Deconinck ${ }^{2}$, I. De Graeve ${ }^{1} \&$ H. Terryn ${ }^{1}$ \\ ${ }^{1}$ Vrije Universiteit Brussel, \\ Department of Metallurgy, Electrochemistry and Material Sciences, \\ Brussel, Belgium \\ ${ }^{2}$ Vrije Universiteit Brussel, Computational Electrochemistry Group, \\ Department of Electrical Engineering and Power Electronics, Brussel, \\ Belgium
}

\begin{abstract}
The anodizing of aluminium is an electrochemical surface treatment yielding the formation of an alumina film, the characteristics of the formed oxide strongly depending on the considered anodizing conditions. Heat transfer has an important influence on the anodizing process, which can be explained by considering the production of heat near the aluminium anode, combined with the significant influence of the local electrode temperature on the process of oxide formation. The influences of temperature and heat transfer on the growth of the anodic oxide film during anodizing of high purity $\mathrm{Al}$ are studied on a laboratory scale in a wall-jet electrode reactor. The impinging jet configuration of the reactor creates a non-uniformly accessible electrode with variable convection as a function of the radial position on the anode. The influence of the resulting non-uniform heat transfer on the local temperature of the electrode is monitored by local temperature measurements on the backside of the aluminium anode, whereas its influence on local film growth is evaluated by means of FEG-SEM surface and cross sectional analyses. A comparison between the simulated and experimentally acquired data is presented. The controlled and known electrolyte flow in the wall-jet reactor enable numerical simulations of the convection which supply additional information on the encountered conditions of heat transfer. The anodizing process itself is simulated using a model based on the high field theory.

Keywords: aluminium, anodizing, heat transfer, wall-jet reactor, modelling.
\end{abstract}




\section{Introduction}

Anodizing of aluminium is a well-known electrochemical process during which an anodic oxide layer is formed on the aluminium anode. The properties of this alumina layer strongly depend on the considered anodizing conditions. When performed in acid electrolytes (e.g. solutions of sulphuric, phosphoric or oxalic acid) it leads to the formation of a porous oxide, which improves, among other properties, corrosion resistance, wear resistance, hardness, compatibility with adhesives and paint of the underlying aluminium. Anodizing in sulphuric acid electrolytes leads to the formation of a porous oxide film with pore diameters and barrier layers generally in the range of 10 to $30 \mathrm{~nm}$ [1]. Products of sulphuric acid anodized aluminium are almost universally used for decorative and protective purposes [1].

Despite the fact that anodizing of aluminium is a widely used and relatively old process, depending on the convection conditions and anodizing geometry industrial anodizers are still confronted with non-uniform oxide thicknesses. This is undesired and might lead to a reduced corrosion protection, local differences in appearance, etc. To avoid these problems reactor optimisation is often based on a trial-and-error approach. Hence from the viewpoint of reactor design simulations, which accurately predict the evolution and outcome of the anodizing process, would certainly be an improvement.

To enable such simulations a model, which correctly describes the anodizing process, is required. The difficulty in the formulation of this model can mainly be attributed to the high sensitivity of the anodizing process to temperature. In contrast to most electrochemical systems in which mass transfer is the determining factor, anodizing of aluminium is a process in which heat transfer needs to be considered. This is due to the fact that on one hand heat is produced during anodizing, the main source being the Joule heating due to ionic current passing through the highly resistive oxide layer on the aluminium electrode. On the other hand the local electrode temperature plays an important role in the process of oxide formation [2,3]. Inadequate removal of the produced heat results in non-uniform films with locally thicker oxide layers [2-4] or in the extreme case even in burning [1-3,5], a local phenomenon which leads to the formation of a region with a very thick but highly degraded oxide layer [1]. Hence, for the modelling of anodizing of aluminium a correct description of the complex relation between current density and (electrode) temperature, in combination with an accurate calculation of the conditions of heat transfer, is a key issue.

For the validation of a model for anodizing the possibility to compare simulated and experimentally measured data is essential. Inevitably these data need to be acquired during anodizing in a set-up with known and controlled convection. In this paper a wall-jet electrode reactor is considered for this purpose. The flow pattern in the wall-jet configuration is due to a submerged fluid jet impinging perpendicularly on the working electrode and spreading out radially, with the fluid outside the jet being at rest [6]. For laminar as well as for turbulent flow the characteristics of the convection are described in literature 
[6-9]. The convective and thermal transfer coefficients are the highest at the centre of the electrode, where the fresh electrolyte impinges on the surface, and decline towards the border of the electrode [7,10,11]. Additionally, information on the influence of the varying local transfer coefficients across the electrode surface on the local electrode temperature is obtained by measurement of local electrode temperatures at different radial positions $[2,3,5]$.

In this paper the use the wall-jet reactor as a tool for the validation of a numerical model for the anodizing of aluminium is described. Experimentally anodizing experiments have been performed under conditions of varying current density and convection. Acquired global and local parameters have been compared with numerically simulated ones.

\section{Experimental}

Disk shaped high purity aluminium samples $(99.99 \%$ Al sheet $0.3 \mathrm{~mm})$, with a diameter of $55 \mathrm{~mm}$ were used, the diameter of the active surface of the anode being $40 \mathrm{~mm}$. Prior to anodizing samples were alkaline etched in $60 \mathrm{~g} / \mathrm{l} \mathrm{NaOH}$ solution at $60^{\circ} \mathrm{C}$ for $60 \mathrm{~s}$, followed by a desmutting treatment consisting out immersion in a $1: 1$ concentrated $\mathrm{HNO}_{3}: \mathrm{H}_{2} \mathrm{O}$ solution at room temperature during 90 s.

The configuration of the wall-jet electrode cell is illustrated in Figure 1. A circular nozzle with diameter $2.0 \mathrm{~mm}$ was used, the distance between the nozzle exit and the working electrode $\mathrm{H}$ was $27 \mathrm{~mm}$. A cylindrical aluminium rod was used as a counter electrode (CE). The distance in height between the $\mathrm{CE}$ and the WE was $80 \mathrm{~mm}$; the distance in height between the RE and the WE was $150 \mathrm{~mm}$. Local temperatures were measured at the backside of the WE by five thermocouples (type T), embedded in the sample holder at 5 different radial positions, these being $-15 \mathrm{~mm},-10 \mathrm{~mm}, 0 \mathrm{~mm},+5 \mathrm{~mm}$ and $+15 \mathrm{~mm}$ from the centre of the anode respectively.

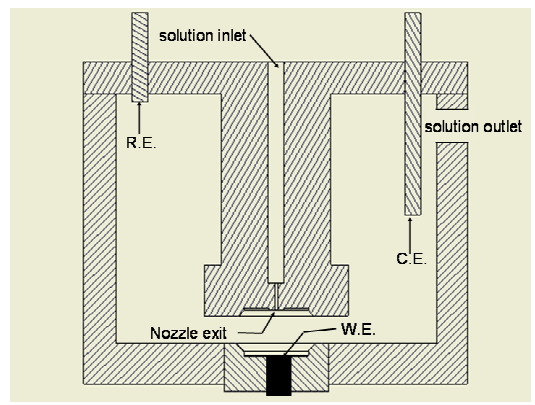

Figure 1: $\quad$ Schematic visualisation of the wall-jet electrode cell [5].

As anodizing electrolyte a $145 \mathrm{~g} / 1 \mathrm{H}_{2} \mathrm{SO}_{4}+5 \mathrm{~g} / 1 \mathrm{Al}_{2}\left(\mathrm{SO}_{4}\right)_{3} .18 \mathrm{H}_{2} \mathrm{O}$ solution at $45.0^{\circ} \mathrm{C}$ was used. Thermostatic control of the electrolyte temperature $\left( \pm 0.1^{\circ} \mathrm{C}\right)$ was ensured by a Lauda RP845 thermostat that controlled the flow of $\mathrm{H}_{2} \mathrm{O}$ 
through a glass heat exchanger immersed in the 451 electrolyte reservoir and which was equipped with an external PT-100 temperature probe.

Anodizing was performed under galvanostatic conditions, the current being supplied by a Delta Elektronica SM 300-20 power source. Two different current densities were applied: $4 \mathrm{~A} / \mathrm{dm}^{2}$ and $8 \mathrm{~A} / \mathrm{dm}^{2}$. A constant charge density of $720 \mathrm{C} / \mathrm{dm}^{2}$ was considered, which corresponded to an anodizing time of $300 \mathrm{~s}$ and $150 \mathrm{~s}$ at $4 \mathrm{~A} / \mathrm{dm}^{2}$ and $8 \mathrm{~A} / \mathrm{dm}^{2}$ respectively. Three different convection conditions were considered: the first at a low flow rate only slightly higher than natural convection, the second within the laminar regime and the third in the turbulent regime. The corresponding Reynolds numbers (based on the nozzle diameter) $R e_{a}$ were 200, 800 and 5000 respectively. For each experimental condition at least 3 measurements were performed. The evolution of the potential of the working electrode (WE) versus a $\mathrm{Ag} / \mathrm{AgCl}$ reference electrode $(\mathrm{RE})(+222 \mathrm{mV}$ vs. NHE at $25^{\circ} \mathrm{C}$ ) was recorded with a National Instruments M-6220 DAQ card and using National Instruments VI-Logger software.

FE-SEM observations were performed using a Jeol JSM-7000F FE-SEM. To avoid charging effects due to the non-conductive properties of the oxide, the surfaces of the samples for FE-SEM analysis were covered with a $1.5 \mathrm{~nm} \mathrm{Pt} / \mathrm{Pd}$ layer, applied by a Cressington 208 HR sputter coater equipped with a Cressington MTM-20 thickness controller. Information on the oxide thickness of the porous films at different radial positions on the anode was acquired by FESEM cross-section images of the oxides at different radial positions. On these images the thickness of the observed cross-sectioned oxide was measured using the Jeol SMile View software.

\section{Anodizing experiments}

\subsection{Results}

Under conditions of varying convection and applied current density several anodizing experiments were performed in the wall-jet reactor. Systematically the evolutions of the anode potential and of the local anode temperatures were recorded. These data, which can be considered as fingerprints of the anodizing process, combined with observations of the formed anodic film, can be used to gain insight on the influence of heat transfer on the process of oxide formation. In Figures 2 mean values of the local anode temperatures after an applied charge density of $1200 \mathrm{C} / \mathrm{dm}^{2}$ (i.e. at the end of the anodizing process) are presented for the different considered flow conditions. In general the temperature of the anode increases with radial distance from the centre up to a radial position of $10 \mathrm{~mm}$, followed by a decrease towards the border of the electrode. This temperature difference between the centre of the anode and a radial position of $10 \mathrm{~mm}$ decreases with increasing $R e_{a}$. At a higher current density of $8 \mathrm{~A} / \mathrm{dm}^{2}$ the differences in local temperatures between the centre and a radial distance of $10 \mathrm{~mm}$ increase. Here local temperature differences are still observed for $R e_{a}=5000$ whereas practically a uniform temperature is recorded at this flow rate at $4 \mathrm{~A} / \mathrm{dm}^{2}$. For all considered anodizing conditions the temperature decreases 
from $10 \mathrm{~mm}$ towards $15 \mathrm{~mm}$ from the centre. Upon applying a current density of $4 \mathrm{~A} / \mathrm{dm}^{2}$ the overall anode temperature increases with increasing $R e_{a}$. This effect is observed on a significantly reduced scale when $8 \mathrm{~A} / \mathrm{dm}^{2}$ is applied.

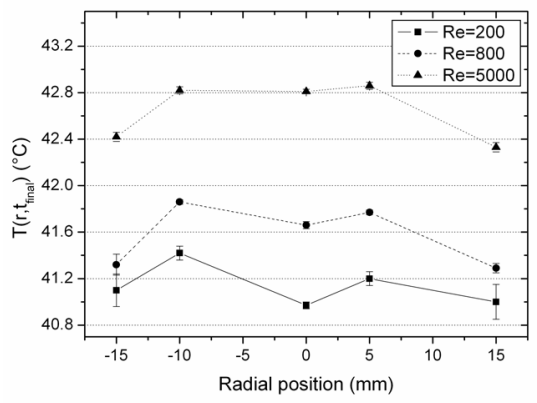

Figure 2(a)

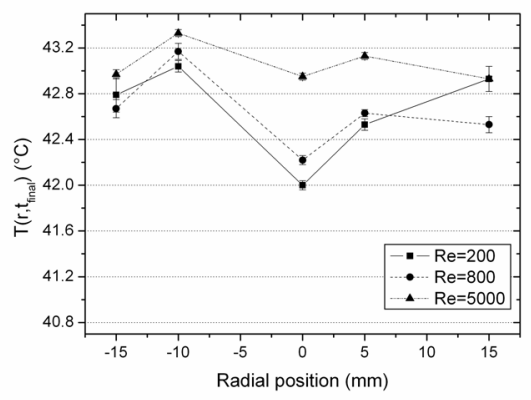

Figure 2(b)

Figure 2: Local anode temperatures after an applied charge density of $1200 \mathrm{C} / \mathrm{dm}^{2}$; (a) applied current density $4 \mathrm{~A} / \mathrm{dm}^{2}$; (b) applied current density $8 \mathrm{~A} / \mathrm{dm}^{2}$.

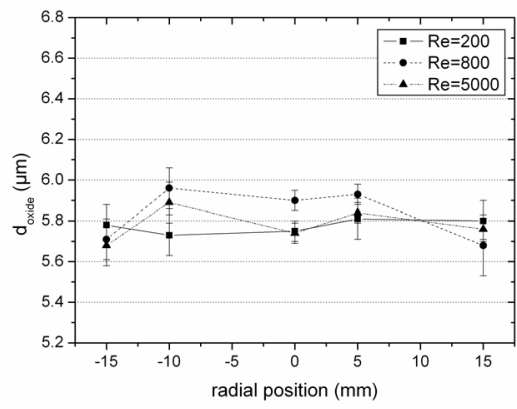

Figure 3(a)

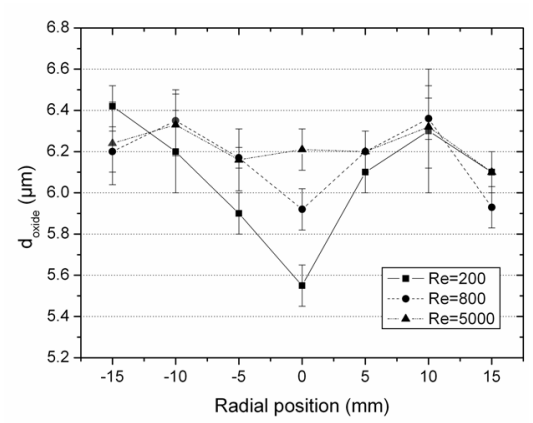

Figure 3(b)

Figure 3: Oxide thickness distribution along electrodes anodized under an applied current density of (a) $4 \mathrm{~A} / \mathrm{dm}^{2}$ and (b) $8 \mathrm{~A} / \mathrm{dm}^{2}$.

The thicknesses of the formed anodic oxide films, determined by crosssectional FE-SEM analyses at different positions on the anodes, are displayed in Figure 3. The distribution of the oxide thickness along the anodes on the samples anodized at $4 \mathrm{~A} / \mathrm{dm}^{2}$ displays slightly the same trend as the corresponding local electrode temperatures. The oxide thickness increases from the centre of the anode up to a radial distance of $10 \mathrm{~mm}$, followed by a decrease towards the edge of the active surface. Though, the observed differences in thickness are relatively moderate and, in contrast to the local temperatures, the local oxide thickness is not directly linked to the flow rate. However, when a current density of $8 \mathrm{~A} / \mathrm{dm}^{2}$ is applied, the convection does significantly influence the local oxide thickness. 
The tendency of an increasing thickness from the centre towards a position of $10 \mathrm{~mm}$, followed by a decrease towards the edge is present under all considered convection conditions and becomes more pronounced when the electrolyte flow is reduced.

\subsection{Discussion}

The moderate differences in local temperatures when anodizing at $4 \mathrm{~A} / \mathrm{dm}^{2}$ indicate that under this condition the amount of heat produced at the anode by the anodizing process can still sufficiently be removed by the convection. Indeed, the observed pronounced influence of the considered flow condition on the overall anode temperature confirms that under these conditions the main heat input is not due to the anodizing reaction but due to heat transfer created by the impinging jet of warm electrolyte. The relatively uniform oxide thicknesses on these samples are consistent with the small differences in local electrode temperatures. As known from literature [2-4] differences in local oxide thickness can directly be linked to differences in local electrode temperatures during anodizing. The differences in overall anode temperatures observed when varying convection are not reflected in different oxide thicknesses. Due to their small scale, in combination with sufficient heat removal by convection and the galvanostatic anodizing conditions, the encountered differences in overall temperature might moderately affect the microstructure of the formed anodic film [12] but will not lead to different overall oxides thicknesses.

At $8 \mathrm{~A} / \mathrm{dm}^{2}$ the effect of varying convection conditions on the overall anode temperature is strongly reduced. Additionally the heat locally produced at the anode by the anodizing process has increased to a level where convective heat removal is no longer sufficient to avoid the occurrence of differences in local temperatures. In this case the local heat generation by anodizing has become more important than heat input due to convection. As indicated above, the different local temperatures are in correspondence with observed variations of local oxide thickness: higher local temperatures will locally induce the growth of thicker films [2-4].

In the next paragraph anodizing experiments under these conditions will be simulated and numerically and experimentally acquired data will be compared.

\section{Numerical simulations}

\subsection{Model}

An overview of the governing equations and boundary conditions used to describe the electrochemical system is presented in [13]. Concerning the fluid dynamics the velocity field $\vec{v}$ and pressure $p$ are calculated in each point of the reactor by solving the Navier-Stokes equation in combination with the conservation of mass for an electrolyte, which is assumed to be incompressible. Additionally, a low-Re $k-\omega$ turbulence model [14] is used to calculate the turbulent viscosity. When the fluid flow field is known the temperature 
distribution in the reactor and along the anode are determined. The local heat dissipation in the fluid and the aluminium due to Ohmic losses are taken into account in the model, but are negligible. On the other hand, for the heat balance of the electrode the heat generated due to anodizing process will be a more important heat source. Furthermore, in calculation of the temperature of the anode the heat loss from the reactor to the surrounding was taken into account. Neglecting concentration gradients in the electrolyte, the electrical potential distribution in the reactor is given by the Laplace equation. This is also the case for the aluminium anode. These equations are solved with the appropriate boundary conditions, of which the electrode boundary conditions for the electrochemical reactions are the most complex. On the cathode still a simple linear behaviour is assumed to describe the hydrogen evolution, though the boundary condition describing the relation between temperature, overpotential and local current density for the anodizing process is much less straightforward. Based on theoretical considerations, the following temperature dependent ButlerVolmer overpotential relation was suggested:

$$
J=D \cdot T^{m} \cdot e^{\frac{B}{T}} \cdot\left(e^{\frac{\alpha z F \eta}{R T}}-e^{\frac{-(1-\alpha) z F \eta}{R T}}\right)
$$

with $D=9.86 \mathrm{E}-6, B=-2600, \alpha=0.0116, z=3$ and $m=0.5$. The values of these parameters were fitted from experimental data in an experimental set-up with limited convection [16].

In the scope of this paper the calculated temperature and local current density distributions along the anode at the end of the anodizing process will be considered. A direct comparison between simulated and measured local temperatures is possible, whereas to come to a similar comparison between simulated and measured values, the local oxide thickness distribution is calculated from the numerically acquired local current density. Based on Faraday's Law, and assuming a constant growth rate, the following expression can be found for the evolution of the oxide thickness $d_{o x}$ with anodizing time:

$$
d_{o x}=\varepsilon \frac{M j \Delta t}{z F \rho}
$$

with $\varepsilon$ the anodizing efficiency, $M$ the molecular weight and $\rho$ the density of the formed of $\mathrm{Al}_{2} \mathrm{O}_{3} . \quad \varepsilon$ was determined based on experimentally observed average oxide thicknesses. The influence of sulphate incorporation into the alumina film on $\varepsilon$ was not taken into account [2]. Under the considered conditions the efficiency was found to vary with the applied current density but to be independent of the considered flow condition. Respective values of 0.85 and 0.90 for applied current densities of $4 \mathrm{~A} / \mathrm{dm}^{2}$ and $8 \mathrm{~A} / \mathrm{dm}^{2}$ were obtained.

\subsection{Results}

Due to the axis-symmetry of the wall-jet reactor the equations mentioned above are solved axis-symmetrically to obtain a full 3D result. A comparison between the simulated and experimentally measured local electrode temperatures from the centre towards the border of the anode is presented in Figure 4. As can be 
observed in all cases too high overall temperatures are calculated. Also an inverted influence of the flow rate on the overall temperatures is found. Whereas experimentally the overall temperature increased with increasing $R e_{a}$, the simulations predict an increasing overall temperature with decreasing $R e_{a}$. Concerning the local temperatures, for an applied current density of $4 \mathrm{~A} / \mathrm{dm}^{2}$ the calculated distributions underestimate the radial increase of the temperature up to $10 \mathrm{~mm}$ from the centre in the case of $R e_{a}=200$ and 800. On the other hand, besides a difference in overall temperature there is a good correspondence between the experimental and calculated temperature evolution at $R e_{a}=5000$. This is also the case when a current density of $8 \mathrm{~A} / \mathrm{dm}^{2}$ is considered. At $8 \mathrm{~A} / \mathrm{dm}^{2}$ in general the radial increase of the local temperature up to a distance of approximately $10 \mathrm{~mm}$ from the centre, followed by a decrease towards the border of the electrode, is numerically also obtained. In contrast to $4 \mathrm{~A} / \mathrm{dm}^{2}$ the simulations indicate an overestimation of the radial temperature increase in the case of $R e_{a}=200$ and 800 .

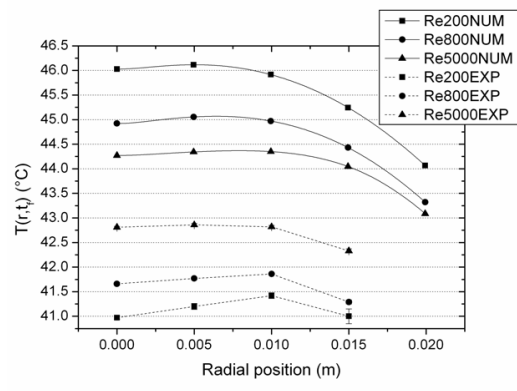

Figure 4(a)

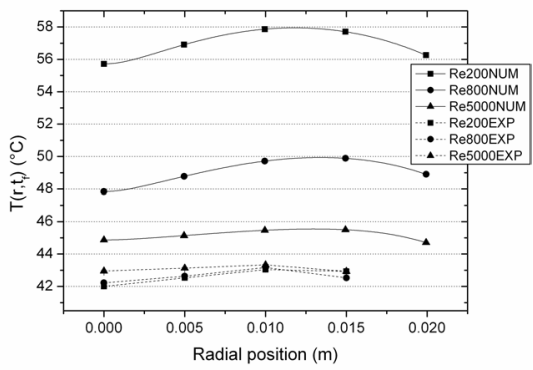

Figure 4(b)

Figure 4: Simulated and experimental local anode temperatures after an applied charge density of $1200 \mathrm{C} / \mathrm{dm}^{2}$. "NUM" and "EXP" respectively indicate numeric and experimental values. Applied current density (a) $4 \mathrm{~A} / \mathrm{dm}^{2}$ and (b) $8 \mathrm{~A} / \mathrm{dm}^{2}$.

Figure 5 displays a comparison between the experimentally determined and numerically calculated evolutions of oxide thickness along the anodes. For both current densities the large variations observed at the centre and at the border of the electrode are due to edge effects occurring during the calculation of the current density and will be neglected in the following discussion. Considering a current density of $4 \mathrm{~A} / \mathrm{dm}^{2}$ the outcome of the numerical simulations is a uniform oxide thickness, whereas experimentally small variations in oxide thickness, in correspondence with variations in local temperature, are observed. Despite the numerical overestimation of the local temperature differences in the case of an applied current density of $8 \mathrm{~A} / \mathrm{dm}^{2}$ and $R e_{a}$ values of 200 and 800 , the model also predicts a too uniform oxide thickness evolution in comparison to the experiments under these conditions. Furthermore, in the case of $R_{a}=200$ at $8 \mathrm{~A} / \mathrm{dm}^{2}$ a higher overall current density and thus overall oxide thickness is calculated due to the significantly overestimated overall temperature. 


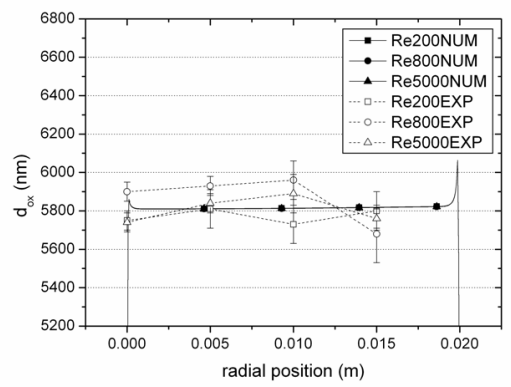

Figure 5(a)

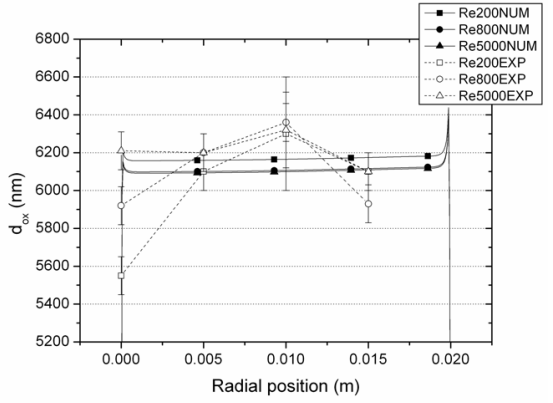

Figure 5(b)

Figure 5: $\quad$ Simulated and experimental evolution of oxide thickness along electrodes. "NUM" and "EXP" respectively indicate numeric and experimental values. Applied current density (a) 4A/dm² and (b) $8 \mathrm{~A} / \mathrm{dm}^{2}$.

\subsection{Discussion}

The generally overestimated overall temperatures and the numerically obtained trend of an increasing overall temperature with decreasing $R e_{a}$ point out that the simulations overestimate the local heat generation of the anodizing process, or that the heat transfer due to the impinging jet is underestimated, or a combination of both. The overestimation of the radial temperature increase in the case of $R e_{a}=200$ and 800 at $8 \mathrm{~A} / \mathrm{dm}^{2}$ can also be explained by considering these effects. On the other hand the underestimated radial temperature increases in the case of $R e_{a}=200$ and 800 at $4 \mathrm{~A} / \mathrm{dm}^{2}$ indicate an overestimated heat loss at the edge of the anode. This is most pronounced under these conditions since in this case the heat input from the anodizing process as well as for the impinging jet of warm electrolyte are the most reduced.

For both considered current densities numerically too uniform evolutions of the local current density and local oxide thickness are obtained. Hence, in the boundary condition describing the anodizing process the local current density is not sensitive enough towards variations in local temperature.

Improvement of numerical simulations will involve the use an updated boundary condition for the anodizing process, better describing the interaction between local current density, local temperature and overpotential. Not only more insight on the point of the anodizing process is necessary, a more advanced description of the heat loss from the reactor to the surrounding is necessary.

\section{Conclusions}

The use of the wall-jet reactor as a tool for the experimental investigation, as well as for the numerical simulation of the influence of heat transfer on the anodizing of aluminium has been demonstrated. The influence of a variation in convection and applied current density on the local electrode temperatures and 
oxide thickness was investigated. Experimentally acquired data were compared with numerical calculated data. To improve accordance between experiments and simulations modifications to and refinement of the used model are necessary.

\section{Acknowledgements}

The authors acknowledge the support from the Instituut voor de aanmoediging van innovatie door Wetenschap \& Technologie in Vlaanderen (IWT, contract nr. SBO 040092).

\section{References}

[1] P.G. Sheasby, R. Pinner, The Surface Treatment and Finishing of Aluminium and its Alloys, 6th Edition, ASM International, USA/ Finishing Publications Ltd, UK, 2001.

[2] I. De Graeve, H. Terryn, G.E. Thompson, J. Electrochem. Soc. 150, p.B158, 2003.

[3] I. De Graeve, H. Terryn, G.E. Thompson, J. Appl. Electrochem. 32, p.73, 2002.

[4] A.J. Bosch, P. Boerstoel, Th. Zuidwijk, A. Hovestad, A. Plomp, J.A. van de Heuvel, ATB Metallurgie, 43, p.65, 2003.

[5] T. Aerts, I. De Graeve, H. Terryn, ATB Metallurgie, in press.

[6] M.B. Glauert, J. Fluid Mech. 1, p.625, 1956.

[7] H. Marin, in Advances in Heat Transfer, Vol.13, ed. by J.P. Hartnett and T.F. Irvine Jr., Academic Press, New York, 1977, p.1-60.

[8] W.J. Albery, C.M.A. Brett, J. Electroanal. Chem. 148, p.211, 1983.

[9] D-T. Chin, C-H. Tsang, J. Electrochem. Soc. 125, p.1461, 1978.

[10] D.J. Bizzak, M.K. Chyu, Int. J. Heat Mass Transf. 38, p.267, 1995.

[11] B. Ellison, B.W. Webb, Int. J. Heat Mass Transf.37, p.1207, 1994.

[12] T. Aerts, Th. Dimogerontakis, I. De Graeve, J. Fransaer, H. Terryn, Surf. Coat. Technol., in press.

[13] G. Nelissen, B. Van Den Bossche, A. Van Theemsche, J. Deconinck, Proc. AESF SUR/FIN, p.386, 2001

[14] D.C. Wilcox, Turbulence modelling for CFD, DCW Industries 2nd edition, 1998.

[15] G. Nelissen, A. Van Theemsche, C. Dan, B. Van den Bossche, J. Deconinck, J. Electroanal. Chem., 563, p.213, 2004.

[16] B. Van den Bossche, J. Deconinck, Proc. BEM 14 Conference, 1992. 